\title{
Generation of a scale-invariant spectrum of adiabatic fluctuations in cosmological models with a contracting phase
}

\author{
Fabio Finelli* \\ Theory Division, CERN, CH-1211 Genève 23, Switzerland \\ and I.A.S.F.- Sezione di Bologna, C.N.R., Via Gobetti 101, 40129 Bologna, Italy \\ Robert Brandenberger ${ }^{\dagger}$ \\ Theory Division, CERN, CH-1211 Genève 23, Switzerland \\ and Department of Physics, Brown University, Providence, Rhode Island 02912
}

(Received 16 January 2002; published 15 May 2002)

\begin{abstract}
In pre-big-bang and in ekpyrotic cosmology, perturbations on cosmological scales today are generated from quantum vacuum fluctuations during a phase when the Universe is contracting (viewed in the Einstein frame). The backgrounds studied to date do not yield a scale-invariant spectrum of adiabatic fluctuations. Here, we present a new contracting background model (neither of pre-big-bang nor of the ekpyrotic form) involving a single scalar field coupled to gravity in which a scale-invariant spectrum of curvature fluctuations and gravitational waves results. The equation of state of this scalar field corresponds to cold matter. We demonstrate that if this contracting phase can be matched via a nonsingular bounce to an expanding Friedmann cosmology, the scale-invariance of the curvature fluctuations is maintained. We also find new background solutions for prebig-bang and for ekpyrotic cosmology, which involve two scalar fields with exponential potentials with background values which are evolving in time. We comment on the difficulty of obtaining a scale-invariant spectrum of adiabatic fluctuations with background solutions which have been studied in the past.
\end{abstract}

DOI: 10.1103/PhysRevD.65.103522

PACS number(s): $98.80 . \mathrm{Cq}$

\section{INTRODUCTION}

Both pre-big-bang (PBB) and ekpyrotic cosmology are attempts to construct alternatives to inflationary cosmology by introducing ideas of string theory to cosmology. The prebig-bang scenario [1] is based on considering the dilaton on an equal footing with the gravitational field, motivated by the fact that these are the important low energy degrees of freedom. The action is given (in the string frame) by

$$
S=\frac{1}{2 \kappa^{2}} \int d^{4} x \sqrt{-g} e^{-\varphi}\left(R-(\partial \varphi)^{2}\right),
$$

where $\varphi$ denotes the dilaton and $\kappa^{2} \equiv M_{\mathrm{pl}}^{-2} \equiv 8 \pi G$. The Universe is assumed to begin in a cold empty state with an accelerating dilaton. The initial evolution is dominated by the effects of the dilaton, and in the string frame yields super-exponential expansion. In the Einstein frame, this corresponds to a contracting phase with a scale factor $a(t)$ given by $a(t) \sim(-t)^{1 / 3}$ (the time $t$ is negative in this phase). By a duality transformation, this solution is related to an expanding Friedmann-Robertson-Walker cosmology. Without corrections to the action (1), however, the initial dilatondominated (PBB) branch and the late time expanding (post BB) branch are separated by a singularity. Since the Hubble radius decreases faster than the physical wavelength corresponding to fixed comoving scales, quantum fluctuations on microscopic scales during the PBB branch can be stretched to scales which are cosmological at the present time, as in the

\footnotetext{
*Email address: finelli@tesre.bo.cnr.it

†Email address: rhb@het.brown.edu
}

case of inflationary cosmology. See, e.g. [2,3] for recent reviews of pre-big-bang cosmology.

The ekpyrotic scenario [4] (see also [5]) assumes that the visible Universe is a boundary brane in five dimensional bulk space-time, and that the heating event which corresponds to the big bang of standard cosmology resulted from the collision of this brane with a parallel one which is attracted to it by an interbrane potential $V(\varphi)$. The dynamics is described by a four-dimensional toy model in which the separation of the branes in the extra dimension is modeled as a scalar field $\varphi$. The effective action is taken to be

$$
S=\int d^{4} x \sqrt{-g}\left(\frac{1}{2 \kappa^{2}} R+\frac{1}{2}(\partial \varphi)^{2}-V(\varphi)\right),
$$

with a potential which for values of $\varphi$ relevant to the generation of cosmological fluctuations is given by

$$
V(\varphi)=-V_{0} e^{-\sqrt{(2 / p)}\left(m_{p}\right)^{-1} \varphi},
$$

where $0<p \ll 1$ and $m_{p}$ denotes the 4D Planck mass (using the notation of [6]). The branes are assumed to start out widely separated and at rest. In this case, the energy is negative and the scale factor associated with the action (2) is contracting with $a(t) \sim(-t)^{p}$ (the time $t$ is again negative in this phase). The time $t=0$ corresponds to a singularity of the four dimensional model (2), as in the case of pre-big-bang cosmology. ${ }^{1}$ As in the case of pre-big-bang cosmology, comoving scales contract less fast than the Hubble radius during this phase, and thus it is again possible that microscopic

\footnotetext{
${ }^{1}$ See, however, Refs. [7-10] for criticism of the scenario.
} 
sub-Hubble scale fluctuations during the phase of contraction produce perturbations on cosmological scales today.

Neither for pre-big-bang cosmology nor in the ekpyrotic scenario $^{2}$ is a scale-invariant spectrum of adiabatic fluctuations generated at the level of the single field actions described above. A heuristic way to understand this is to note that the initial values of the fluctuations when they exit the Hubble radius are set by the Hubble constant. The Hubble constant is increasing rapidly as a function of time in both scenarios, and thus a deeply blue spectrum of initial fluctuations will result (spectral index $n=4$ in the case of pre-bigbang cosmology, $n=3$ in the case of the ekpyrotic scenario). Careful studies taking into account the gravitational dynamics on super-Hubble scales confirmed this result both for prebig-bang cosmology [12] and in the ekpyrotic scenario $[6,13-16]^{3}$

The idea of obtaining the "big bang" of our Universe from a previous phase of cosmological contraction is, however, very interesting. In Sec. II we discuss a model consisting of scalar field matter with an equation of state $P=0, P$ denoting pressure, obtained with the value $p=2 / 3$ for the exponential potential in Eq. (3) (we note that for $p=2 / 3$ the potential is positive, i.e. $V_{0}<0$, as opposed to the case of the ekpyrotic model [4], where the potential is negative, i.e. $V_{0}$ $>0$ ). In this background, the quantum vacuum fluctuations of the field during the phase of contraction, matched to an expanding Friedmann cosmology at a nonsingular bounce, yield a scale-invariant spectrum of curvature fluctuations. ${ }^{4}$ Such a model is obtained here by considering an exponential potential for the scalar field. In the PBB scenario exponential potentials for the dilaton may be generated by nonperturbative effects or by considering non-critical string theory (a cosmological constant in the string frame can generate an exponential potential in the Einstein frame).

In order to connect the contracting phase to an expanding phase, it is necessary to assume that at sufficiently high curvatures corrections to Einstein gravity become important, yielding a nonsingular bounce. Similar ideas are invoked to achieve a graceful exit in pre-big-bang cosmology. In Sec. III we apply matching conditions $[20,21]$ corresponding to continuity of the induced metric and of the extrinsic curvature for the infrared modes to calculate the induced curvature fluctuations in the expanding phase. We find that the dominant mode of the curvature perturbation $\zeta$ in the expanding phase inherits the scale invariance of the growing mode of

\footnotetext{
${ }^{2}$ Note that the fluctuation generation mechanism in the recently proposed cyclic model [11] is the same as in the ekpyrotic scenario, and hence also does not yield a scale-invariant spectrum of fluctuations.

${ }^{3}$ In the case of the ekpyrotic scenario, the calculation of [17] yields a different result, but at least in our opinion it is flawed because it is based on an ad hoc matching condition at the bounce which (as already mentioned in [13]) does not yield the correct result when applied to power-law inflation (see also [18] and [19] for recent work on this issue).

${ }^{4}$ Note that we are assuming the absence of any initial classical fluctuations, as is done in the ekpyrotic scenario.
}

the contracting phase. A new aspect of this matching problem is that the dominant mode of $\zeta$ increases on superHubble scales in the contracting phase, in contrast to what occurs in inflationary cosmology, where it is constant.

In Sec. IV we study backgrounds with two scalar matter fields. Note that both for pre-big-bang cosmology and in the ekpyrotic scenario, there are other light fields which should be included in the respective actions (1) and (2). In both cases there are axion and moduli fields which could play an important role. These fields can be dynamical during the collapse phase [22].

In the case of pre-big-bang cosmology, it was realized in [23] that, in the presence of moving extra dimensions, axion fluctuations are amplified, and that the motion of the extra dimensions can be chosen such that a scale invariant spectrum of isocurvature perturbations results (for more work along these lines see e.g. $[24,25])$. However, such a primordial spectrum of inhomogeneities seeded by axion fluctuations is ruled out by the latest CMB anisotropy results (see e.g. [25] for an analysis of this issue).

In Sec. IV of this paper, we present a new class of two scalar field backgrounds. These backgrounds have an interpretation both in the case of the pre-big-bang scenario and in ekpyrotic cosmology. In the former case they correspond to a dilaton-axion background with exponential dilaton potential, in the latter case they involve the scalar field $\varphi$ representing the separation of the two branes and a rolling axion.

\section{FLUCTUATIONS IN SINGLE FIELD BACKGROUND MODELS}

We begin with a brief review of the analysis of the spectrum of cosmological fluctuations in single field background models.

To linear order in fluctuations (and neglecting gravitational waves and vector modes), the metric can be written as (see e.g. [26] for a comprehensive review)

$$
d s^{2}=a^{2}(\eta)\left[(1+2 \Phi) d \eta^{2}-(1-2 \Phi) d x^{i} d x_{i}\right],
$$

where $\eta$ is conformal time. We have used the fact that if matter consists of scalar fields there is to linear order no anisotropic stress.

Via the Einstein constraint equations, the linear gravitational fluctuations (described by $\Phi$ ) are coupled to the matter field fluctuations. In the single matter field case, with matter fluctuations denoted by $\delta \varphi$, a convenient and gaugeinvariant variable is [27] (see also [28])

$$
v=a\left(\delta \varphi+\frac{\dot{\varphi}}{H} \Phi\right) \equiv a Q,
$$

with an overdot denoting the derivative with respect to physical time $t$. In particular, in the action for joint metric and matter fluctuations, $v$ is a canonically normalized field, and hence it is useful to quantize the fluctuations in terms of it. We will be studying the linear perturbation equations for this variable in momentum space, with $k$ standing for comoving momentum. 
In an expanding Universe described by the Einstein equations, a convenient variable to use to track the amplitude of the fluctuations on super-Hubble scales is $\zeta$, the curvature perturbation in comoving gauge $[29,30]$. In variable $\zeta$ is related to the metric fluctuations via

$$
\zeta=\frac{2}{3} \frac{\Phi+H^{-1} \dot{\Phi}}{1+w}+\Phi
$$

and it is related to $Q$ and $v$ as follows:

$$
\zeta=\frac{H}{\dot{\phi}} Q=\frac{v}{z},
$$

with

$$
z=a \frac{\dot{\varphi}}{H}
$$

In an expanding Universe and in the absence of entropy fluctuations, $\zeta$ is constant on scales larger than the Hubble radius, as can be seen from its equation of motion which is

$$
\dot{\zeta}=-\frac{H}{\dot{H}} \frac{k^{2}}{a^{2}} \Phi .
$$

In inflationary cosmology, it is thus useful to calculate the magnitude of $\zeta$ at the time when the fluctuation scale becomes larger than the Hubble radius, to use the constancy of $\zeta$ to evolve until the time when the scale re-enters the Hubble radius, and to infer the values of $\Phi$ and $\dot{\Phi}$ (which determine, for example, the spectrum of CMB anisotropies) at that time.

However, in the case of a contracting Universe one must (even in the absence of entropy fluctuations) be more careful, since the term in Eq. (9) proportional to $k^{2}$ may grow. In the case of pre-big-bang cosmology, the growth is only logarithmic in $\eta$, and for the potential used in the original version of the ekpyrotic model there is no growth of $\zeta$ at all. Hence, in these models $\zeta$ remains a good variable to follow the magnitude of the density fluctuations on super-Hubble scales.

In the Einstein frame, the equations of motion for cosmological perturbations reduce to the following equation for the Fourier mode of the variable $v$ defined in Eq. (5) with comoving wave number $k$ (we suppress the index $k$ on $v$ ):

$$
v^{\prime \prime}+\left(k^{2}-\frac{z^{\prime \prime}}{z}\right) v=0
$$

where a prime denotes the derivative with respect to conformal time $\eta$.

Making use of the background equations of motion, this equation becomes

$$
v^{\prime \prime}+\left(k^{2}-\frac{a^{\prime \prime}}{a}+a^{2} V^{\prime \prime}+2 a^{2}\left[\frac{\dot{H}}{H}+3 H\right]\right) v=0,
$$

where $V$ is the potential of the matter field $\varphi$. From this equation it is clear that on scales much smaller than the
Hubble radius, $v$ is oscillating with frequency given by $k$. The vacuum state normalization of $v$ on these scales is

$$
v=\frac{1}{\sqrt{2 k}} e^{-i k \eta}
$$

We consider backgrounds which correspond in the Einstein frame to power law contraction

$$
a(t) \propto(-t)^{p} .
$$

In this case, the last two terms within the parentheses multiplying $v$ in Eq. (11) cancel, and the equation reduces to

$$
v^{\prime \prime}+\left(k^{2}-\frac{p(2 p-1)}{(p-1)^{2}} \frac{1}{\eta^{2}}\right) v=0 .
$$

Its solution can be expressed in terms of Bessel functions $Z_{\nu}$ :

$$
v=\frac{\sqrt{-\pi \eta}}{2} Z_{|\nu|}(-k \eta)
$$

where the index $\nu$ is related to the index $p$ by

$$
\frac{\nu^{2}-1}{4}=\frac{p(2 p-1)}{(p-1)^{2}}
$$

and therefore

$$
\nu=\frac{1}{2} \frac{1-3 p}{1-p}
$$

As can be seen from the large argument expansion of the Bessel functions, this solution automatically has the correct vacuum normalization.

Making use of the long wavelength limit of the Bessel functions, and of the fact that for our class of backgrounds $\dot{\varphi} / H$ is independent of time, we obtain the following power spectrum of the variable $\zeta$ :

$$
P_{\zeta}(k)=\frac{k^{3}}{2 \pi^{2}}|\zeta|^{2} \sim p k^{3-2|\nu|} .
$$

Thus, to obtain a scale-invariant spectrum of adiabatic fluctuations, we require $|\nu|=3 / 2$, whereas in the single field ekpyrotic scenario with $p \sim 0$ one obtains $\nu \sim 1 / 2$ and thus spectral index $n \sim 3$. In the single field pre-big-bang scenario the resulting values are $p=1 / 3, \nu=0$ and therefore $n=4$.

An interesting background is obtained if $p=2 / 3$. In this case, $|\nu|=3 / 2$ and hence a scale-invariant spectrum of adiabatic curvature fluctuations is generated in the collapsing phase..$^{5}$ This background corresponds to a contracting Uni-

\footnotetext{
${ }^{5}$ This result was already noted in the work of [31], and more recently in [13] (it can also be seen from Fig. 3 of [16]). In the earlier work [31], however, the transition to an expanding phase was not discussed.
} 
verse dominated by cold matter with equation of state $P$ $=0$ (with $P$ denoting pressure). The cold matter in this case is modeled by a scalar field with an exponential potential. Note that the fluctuations in this model behave differently than the fluctuations in a hydrodynamical model with $P$ $=c_{s}^{2}=0$, where $c_{s}^{2}$ is the speed of sound. This can be seen by comparing the equation of motion (11) for scalar-fieldinduced fluctuations with the corresponding equation for hydrodynamical matter (see Chap. 5 of [26]). Note that modeling cold matter by a scalar field with nontrivial potential will avoid the Jeans instability problem on small scales which plagues $P=0$ hydrodynamical matter. Note also that such a contraction $\left[a(t) \sim(-t)^{2 / 3}\right]$ solves the horizon problem.

\section{SPECTRUM OF FLUCTUATIONS AFTER THE BOUNCE}

Let us consider the contracting model with $p=2 / 3$ introduced above and imagine that it is connected via a nonsingular bounce to an expanding Friedmann-Robertson-Walker cosmology. In the following we will argue that in such a model the scale-invariant spectrum of $\zeta$ connects through the bounce to a late time scale-invariant spectrum in the expanding phase. Thus, our model yields an alternative to cosmological inflation in providing a mechanism for producing a scale-invariant spectrum of adiabatic fluctuations on scales which could, provided that the phase of contraction lasts sufficiently long, be of cosmological interest today.

The graceful exit problem faced in order to obtain such a bounce is similar to what is required to obtain a graceful exit in pre-big-bang cosmology. In the latter case, there are indications that higher curvature and string loop effects can yield a nonsingular bounce [32-36]. (See also [37,38].) Backreaction effects also can play an important role [39]. There is also a construction $[40,41]$ based on a Lagrangian with higher order corrections of the nonsingular Universe construction $[42,43]$ which yields such a regular bounce. Since the corrections to Einstein gravity will be important only very close to the bounce, and since we are interested in scales which at the bounce are much larger than the Hubble radius, it appears reasonable to model the bounce (for the purpose of matching the pre-bounce and post-bounce fluctuations) as a gluing of two Einstein universes at a fixed surface specified by some physical criterion (see, however, the concerns raised in $[15,18]$ on this issue).

The naive expectation is that the dominant mode of the curvature fluctuation $\zeta$ after the bounce (which is constant in time) is given by the dominant mode prior to the bounce, the mode which in our background has a scale-invariant spectrum. This is what happens in inflationary cosmology with reheating modeled as a discontinuous change in the equation of state $[44,45]$, pre-big-bang cosmology [21] and in the ekpyrotic scenario with a nonsingular bounce [13]. Note, however, that a similar "plausibility" argument applied to the variable $\Phi$ fails in both of the above cases. In both pre-bigbang and ekpyrotic cosmology the pre-bounce growing mode of $\Phi$ does not contribute to leading order in the wave number $k$ to the dominant post-bounce mode of $\Phi$. Thus, in order to be able to draw conclusions about the post-bounce spectrum, it is necessary to carefully match the fluctuation variables at the bounce.

The general relativistic matching conditions demand that at the boundary surface the induced surface metric and the extrinsic curvature be continuous [20,21]. As a matching surface one can choose either a constant energy density surface (from the point of view of longitudinal gauge) or a constant scalar field surface. ${ }^{6}$ For super-Hubble scale fluctuations, the difference between these two surfaces is of order $k^{2}$ and does not affect the results to leading order in $k^{2}$. The matching on a constant energy density surface implies the continuity of $\zeta$ and $\Phi$ across the surface. In pre-big-bang cosmology [21] and in the ekpyrotic scenario [13] this leads to the conclusion that the growing mode of $\Phi$ during the collapse phase (which in the case of the ekpyrotic scenario has a scaleinvariant spectrum) does not couple to leading order in $k^{2}$ to the dominant (constant) mode of the post-bounce phase. In contrast, the late time value of $\zeta$ is the same as at the bounce. These results are confirmed in studies of cosmological perturbations in generalized Einstein theories [16,46,47] which yield a bounce (see also $[48,49]$ ).

At the end of Sec. II we have shown that, for our new background, the growing mode of $\zeta$ in the contracting phase obtains a scale-invariant spectrum. We need to show that to leading order in $k^{2}$ there is a non-vanishing coupling between the pre-bounce growing mode of $\zeta$ and the postbounce dominant mode. Note that the rapid growth of $\zeta$ is very different from what occurs in the inflationary Universe, in pre-big-bang cosmology and in the ekpyrotic scenario, and that the analysis of the matching conditions needs to be reconsidered.

We begin with the following general solution (to leading order in $k^{2}$ ) for $\zeta$ [see e.g. Eq. (12.27) in [26]]:

$$
\zeta=D+S \int \frac{d \eta}{z^{2}},
$$

where $D$ and $S$ are constant coefficients. In our background, the $S$ mode is the growing one, whereas in inflationary cosmology it is decaying and thus subdominant. Making use of Eq. (9), we can find the corresponding form of $\Phi$ :

$$
\Phi=-\frac{1}{2 m_{p}^{2}} \frac{\mathcal{H}}{a^{2}}\left(\frac{S}{k^{2}}\right),
$$

where $\mathcal{H}$ is the Hubble constant in conformal time. This gives the mode of $\Phi$ which is decaying in inflationary cosmology. It also shows that this "decaying" mode of $\Phi$ affects the value of $\zeta$ to order $k^{2}$ (to leading order it cancels out). There is also a constant mode of $\Phi$ which determines the constant mode ( $D$ mode) of $\zeta$. The coefficients of the constant modes of $\Phi$ and $\zeta$ are related by a function of the equation of state of the background which contains no $K$ dependence. Thus, in order to perform the matching analysis we consider the following forms for $\Phi$ and $\zeta$ :

\footnotetext{
${ }^{6}$ See [18] for a justification of this choice of the matching surface.
} 


$$
\begin{gathered}
\zeta=D+S f(\eta), \\
\Phi=\alpha D+\beta \frac{S}{k^{2}} \frac{\mathcal{H}}{a^{2}},
\end{gathered}
$$

where $f(\eta)=\int\left(d \eta / z^{2}\right)$ and where the coefficients $\alpha$ and $\beta$ depend only on the equation of state.

It is now straightforward to calculate the consequences of the continuous matching of $\Phi$ and $\zeta$ across the bounce for the coefficients of the two modes of $\zeta$ before and after the bounce. Quantities before the bounce will be denoted by a superscript - and those after the bounce by a superscript + . Simple algebra yields

$$
\begin{aligned}
D^{+}\left(1-\frac{\alpha^{+} f^{+} a^{2} k^{2}}{\beta^{+} \mathcal{H}^{+}}\right)= & D^{-}\left(1-\frac{\alpha^{-} f^{+} a^{2} k^{2}}{\beta^{+} \mathcal{H}^{+}}\right) \\
& +S^{-}\left(f^{-}-\frac{\beta^{-}}{\beta^{+}} \frac{\mathcal{H}^{-}}{\mathcal{H}^{+}} f^{+}\right) .
\end{aligned}
$$

In the ekpyrotic scenario, it follows from Eq. (15) and from the fact that $\nu=1 / 2$ that $D^{-} \sim k^{-1 / 2}$ and $S^{-} \sim k^{1 / 2}$. Hence, the $D$ mode of $\zeta$ after the bounce has spectral index $n=3$, in agreement with our earlier matching results [13]. However, for our present background we have $\nu=3 / 2$ and hence $D^{-} \sim k^{3 / 2}$ and $S^{-} \sim k^{-3 / 2}$. Since the matching of the $S^{-}$mode to the $D^{+}$mode is not suppressed by factors of $k^{2}$, the post-bounce $D^{+}$mode inherits the scale invariance of the pre-bounce $S^{-}$mode. Thus, we have shown that our background yields a scale-invariant spectrum of fluctuations at late times.

Let us now return to the analysis of Sec. II and determine the amplitude of the scale-invariant spectrum of curvature perturbations at (and, as we have shown above, therefore also after) the bounce in our model with $p=2 / 3$. As follows from Eq. (7) and from the background values of $\dot{\varphi}$ and $H$ :

$$
\zeta=\frac{H}{\dot{\varphi}} Q=\sqrt{\frac{p}{2}} \frac{Q}{M_{\mathrm{pl}}}
$$

According to Eq. (15), the normalized solution for $Q$ is

$$
Q=e^{[i(\tilde{\nu}+1 / 2) \pi / 2]} \frac{\sqrt{-\pi \eta}}{2 a} H_{\nu}(-k \eta)
$$

where $H$ denotes the Hankel function, $\widetilde{\nu}=|\nu|$, and where the scale factor is expressed in terms of conformal time as

$$
a(\eta)=\left(-(1-p) M_{\mathrm{pl}} \eta\right)^{p /(1-p)}
$$

By inserting the long-wavelength limit $(-k \eta \ll 1)$ of the Hankel function one gets
$P_{\zeta}(k)=\frac{k^{3}}{2 \pi^{2}}|\zeta|^{2}=p 2^{2 \tilde{\nu}-3} \frac{\Gamma(\tilde{\nu})^{2}}{2 \pi^{3}}\left[\frac{\mathcal{H}(1-p)}{M_{\mathrm{pl}} p}\right]^{4 \tilde{\nu}} \frac{M_{\mathrm{pl}}^{2 \tilde{\nu}-3}}{k^{2 \tilde{\nu}-3}}$.

For $p=2 / 3$ one has an amplitude in agreement with observations for $\mathcal{H}_{*} / M_{\mathrm{pl}} \sim 10^{-1}$, where $\mathcal{H}_{*}$ indicates the absolute value of the Hubble rate at the bounce (when the contraction stops).

The model with $p=2 / 3$ which we are considering also generates a scale-invariant spectrum of gravitational waves, as already realized in [50] and [31]. This can be seen immediately since $\widetilde{h}=a h$, where $h$ is the amplitude of the tensor perturbation of the metric, obeys the same equation of motion as the scalar fluctuation variable $v$. This is true in all models with an exponential potential for the scalar field [51]. In order to compare the amplitudes of the power spectra $P_{\zeta}$ for scalar metric fluctuations and $P_{h}$ for gravitational waves in the collapsing phase, we first need to divide $v$ by $M_{p l}$ (for dimensional reasons). Then, using the relation (7) between $\zeta$ and $v$ and the background values of $H$ and $\dot{\varphi}$ we immediately obtain

$$
\frac{P_{\zeta}}{P_{h}}=\frac{p}{2}
$$

This is a definite prediction resulting from the analysis during the contracting phase.

\section{A BACKGROUND WITH TWO EVOLVING FIELDS}

We now turn to the question of whether it might be possible to generate a scale-invariant spectrum of adiabatic fluctuations in pre-big-bang cosmology and in the ekpyrotic scenario by turning on nontrivial background time dependence of a suitably chosen second scalar field. As a first step, we will have to construct new background solutions. We will consider the action

$$
S=\int d^{4} x \sqrt{-g}\left(\frac{1}{2 \kappa^{2}} R+\frac{e^{\alpha \varphi / M_{\mathrm{pl}}}}{2}(\partial \sigma)^{2}+\frac{1}{2}(\partial \varphi)^{2}-V(\varphi)\right),
$$

with an exponential potential for $\varphi$ :

$$
V(\varphi)=-V_{0} e^{-\beta \varphi / M_{\mathrm{pl}}}
$$

In the string-inspired scenario, the interpretation of this action is that we add to the single field action (2) a second field $\sigma$ with a non-minimal kinetic term. Such $\sigma$ can be an axionlike field in a modified pre-big-bang scenario where the dilaton $\varphi$ has an exponential potential. In the ekpyrotic scenario, with $\varphi$ representing the separation of the branes, $\sigma$ can be an axion-like field or a second scalar field in an effective theory for the brane worlds.

We now look for self-consistent analytical solutions of the background equations: 


$$
\begin{gathered}
\mathcal{H}^{2}=\frac{1}{3 M_{\mathrm{pl}}^{2}}\left(\frac{\varphi^{\prime 2}}{2}+a^{2} V(\varphi)+e^{\alpha \varphi / M_{\mathrm{pl}}} \frac{\sigma^{\prime 2}}{2}\right) \\
\varphi^{\prime \prime}+2 \mathcal{H} \varphi^{\prime}+a^{2} V_{\varphi}=\frac{\alpha}{2 M_{\mathrm{pl}}} e^{\alpha \varphi / M_{\mathrm{pl}}} \sigma^{\prime 2} \\
\sigma^{\prime \prime}+\left(2 \mathcal{H}+\alpha \frac{\varphi^{\prime}}{M_{\mathrm{pl}}}\right) \sigma^{\prime}=0 .
\end{gathered}
$$

We consider the following ansatz for $a(\eta)$ and $\varphi(\eta)$ :

$$
\begin{aligned}
& a(\eta)=\left(-M_{\mathrm{pl}}(1-p) \eta\right)^{p /(1-p)} \\
& \varphi(\eta)=A \log \left(-M_{\mathrm{pl}}(1-p) \eta\right) .
\end{aligned}
$$

Equation (32) can be immediately integrated giving

$$
\sigma^{\prime}=C \frac{e^{-\alpha \varphi / M_{\mathrm{pl}}}}{a^{2}}
$$

where $C$ is an integration constant. We have the following parameters: $\alpha, \beta, p, A, C$. By imposing that all the terms in the equation for $\varphi$ (31) have the same time dependence we obtain (assuming $V_{0}$ and $C$ are nonzero)

$$
\begin{gathered}
\beta \frac{A}{M_{\mathrm{pl}}}-\frac{2 p}{1-p}=2 \\
\alpha \frac{A}{M_{\mathrm{pl}}}+\frac{4 p}{1-p}=2
\end{gathered}
$$

and these relations lead to the constraint

$$
\alpha / \beta=1-3 p
$$

Note that this relation implies that for the case of pre-bigbang cosmology with $p=1 / 3$ our action describes a modulus field $\sigma$ (with a standard kinetic term).

From the equation for $\mathcal{H}^{\prime},{ }^{7}$

$$
\mathcal{H}^{\prime}-\mathcal{H}^{2}=-\frac{1}{2 M_{\mathrm{pl}}^{2}}\left[\varphi^{\prime 2}+\sigma^{\prime 2} e^{\alpha \varphi / M_{\mathrm{pl}}}\right]
$$

we get

$$
\left(\frac{A}{M_{\mathrm{pl}}}\right)^{2}+\left(\frac{C}{M_{\mathrm{pl}}^{2}(1-p)}\right)^{2}=\frac{2 p}{(1-p)^{2}} .
$$

The interpretation of this result is that $A / M_{p l}$ and $C /\left[M_{p l}^{2}(1-p)\right]$ are constrained to be on a circle of radius proportional to $\sqrt{p}$. From the energy constraint (30) we obtain

\footnotetext{
${ }^{7}$ It is not an independent equation, but is obtained from the time derivative of the Hamiltonian constraint (30), making use of the equations of motion for $\varphi$ and $\sigma$. From this equation it is easy to derive another constraint among the parameters.
}

$$
-\frac{V_{0}}{M_{\mathrm{pl}}^{4}}=p(3 p-1)
$$

This concludes all of the independent relations between the parameters and integration constants for the exact solution for the background obtained from the ansatz (34), (33).

Let us compare the dependence of $a$ and $V(\varphi)$ on $p$ and on time in our two field solution with the corresponding scalings in the single field model of Sec. II. Comparing Eq. (33) with Eq. (25) it follows that the dependence of $a$ on $p$ and on time is the same. Inserting Eq. (34) into Eq. (29), making use of Eq. (41), and comparing with the result in the single field case [see e.g. Eq. (12) of [13]] it follows that the dependence of $V(\varphi)$ on $p$ and on time is also identical. This implies that the dependence on $p$ of the spectrum and amplitude of gravitational waves is the same in the single field and multifield models. Equation (40) shows that the sum of the kinetic terms in the multifield model with the axion is the same as in the single field model.

A final remark for the case $\alpha=0$, in which $\sigma$ corresponds to a modulus field. The solution obeying the ansatz (34) constructed in this section has a meaning for $\alpha=0$, only in the case $p=1 / 3$, i.e. vanishing potential for $\varphi$. Only in this case analytic solutions exist, since both $\varphi$ and $\sigma$ are massless, and the global equation of state is stiff matter (pressure density equal to energy density). In the case of an exponential potential as in Eq. (29), the modulus $\sigma$ dominates the energy density for early times if $p<1 / 3$ and for late times if $p$ $>1 / 3$.

\section{DISCUSSION}

In this paper we have discussed ways of obtaining a scaleinvariant spectrum of adiabatic fluctuations in models in which a contracting Universe is matched via a nonsingular bounce to an expanding Friedmann-Robertson-Walker cosmology. We have assumed that new physics at high curvatures leads to a short period in which the background evolution is not described by the Einstein equations, thus enabling a transition from a contracting to an expanding phase (from the point of view of the Einstein frame scale factor).

Our first result is that a contracting Universe dominated by cold matter modeled as a scalar field with an exponential potential with appropriately chosen index can yield a scaleinvariant spectrum of curvature fluctuations (fluctuations in the variable $\zeta$ ) in the contracting phase, which is matched at the bounce to a scale-invariant spectrum during the expanding phase.

Our second result is that it is possible in the context of ekpyrotic cosmology (and also of other models with a phase of power law contraction with power $p \neq 1 / 3$ ) to obtain new background solutions by considering a model with two scalar fields $\varphi$ and $\sigma$, with exponential potential for $\varphi$ and nonstandard kinetic term coupling for $\sigma$, provided that the coefficients in the exponents of the potential and describing the non-minimal kinetic term satisfy a particular relation. In the context of ekpyrotic cosmology, this corresponds to adding a rolling second field $\sigma$ (an axion-like or the modulus) to the 
usual four-dimensional effective action which contains a scalar field $\varphi$ describing the separation of the branes. It is important that both fields have time-dependent backgrounds. In the context of the pre-big-bang scenario, our model can be interpreted as adding an axionic field to the dilaton-gravity action, and assuming that there is an exponential potential for the dilaton.

Adding the new degree of freedom via the second scalar field leads to an isocurvature mode of the fluctuations. In the PBB scenario, it was shown [23] that for suitable field backgrounds this isocurvature mode has a scale invariant spectrum. However, because of the absence of a potential, the isocurvature and adiabatic modes are not coupled and a spectrum seeded by isocurvature axion fluctuations seems in conflict with observations [25]. In our backgrounds, adiabatic and isocurvature fluctuations are coupled and it might be possible to induce directly a scale invariant spectrum of adiabatic fluctuations.
This mechanism is an alternative to the one proposed recently based on axion decay to obtain a scale-invariant spectrum of adiabatic curvature fluctuations [52-54]. One starts with a scale-invariant spectrum of isocurvature fluctuations (like in the work of [23]), and assumes that the axion field which is responsible for the scale-invariant spectrum decays at some late time when it dominates the background energy density.

\section{ACKNOWLEDGMENTS}

We wish to thank D. Wands for useful correspondence and T. Riotto for interesting comments. This research was supported in part by the U.S. Department of Energy under Contract DE-FG02-91ER40688, TASK A (R.B., Brown University). Both authors thank the TH Division at CERN for hospitality during the course of this work.
[1] M. Gasperini and G. Veneziano, Astropart. Phys. 1, 317 (1993).

[2] G. Veneziano, hep-th/0002094.

[3] J.E. Lidsey, D. Wands, and E.J. Copeland, Phys. Rep. 337, 343 (2000).

[4] J. Khoury, B.A. Ovrut, P.J. Steinhardt, and N. Turok, Phys. Rev. D 64, 123522 (2001).

[5] J. Khoury, B. A. Ovrut, N. Seiberg, P. J. Steinhardt, and N. Turok, Phys. Rev. D 65, 086007 (2002).

[6] D.H. Lyth, Phys. Lett. B 524, 1 (2002).

[7] R. Kallosh, L. Kofman, and A.D. Linde, Phys. Rev. D 64, 123523 (2001).

[8] R. Kallosh, L. Kofman, A.D. Linde, and A.A. Tseytlin, Phys. Rev. D 64, 123524 (2001).

[9] K. Enqvist, E. Keski-Vakkuri, and S. Rasanen, Nucl. Phys. B614, 388 (2001).

[10] S. Rasanen, Nucl. Phys. B626, 183 (2002).

[11] P.J. Steinhardt and N. Turok, hep-th/0111098.

[12] R. Brustein, M. Gasperini, M. Giovannini, V.F. Mukhanov, and G. Veneziano, Phys. Rev. D 51, 6744 (1995).

[13] R. Brandenberger and F. Finelli, J. High Energy Phys. 11, 056 (2001).

[14] J.C. Hwang, Phys. Rev. D 65, 063514 (2002).

[15] D.H. Lyth, Phys. Lett. B 526, 173 (2002).

[16] S. Tsujikawa, Phys. Lett. B 526, 179 (2002).

[17] J. Khoury, B.A. Ovrut, P.J. Steinhardt, and N. Turok, hep-th/0109050.

[18] J. Martin, P. Peter, N. Pinto Neto, and D.J. Schwarz, hep-th/0112128.

[19] R. Durrer, hep-th/0112026.

[20] J. Hwang and E.T. Vishniac, Astrophys. J. 382, 363 (1991).

[21] N. Deruelle and V.F. Mukhanov, Phys. Rev. D 52, 5549 (1995).

[22] E.J. Copeland, A. Lahiri, and D. Wands, Phys. Rev. D 50, 4868 (1994).

[23] E.J. Copeland, R. Easther, and D. Wands, Phys. Rev. D 56, 874 (1997).

[24] R. Durrer, M. Gasperini, M. Sakellariadou, and G. Veneziano,
Phys. Rev. D 59, 043511 (1999).

[25] A. Melchiorri, F. Vernizzi, R. Durrer, and G. Veneziano, Phys. Rev. Lett. 83, 4464 (1999).

[26] V.F. Mukhanov, H.A. Feldman and R.H. Brandenberger, Phys. Rep. 215, 203 (1992).

[27] V.F. Mukhanov, Zh. Éksp. Teor. Fiz. 94N7, 1 (1988) [Sov. Phys. JETP 67, 1297 (1988)].

[28] V. Lukash, Sov. Phys. JETP 52, 807 (1980).

[29] J.M. Bardeen, Phys. Rev. D 22, 1882 (1980).

[30] D.H. Lyth, Phys. Rev. D 31, 1792 (1985).

[31] D. Wands, Phys. Rev. D 60, 023507 (1999).

[32] M. Gasperini, M. Maggiore, and G. Veneziano, Nucl. Phys. B494, 315 (1997).

[33] M. Maggiore and A. Riotto, Nucl. Phys. B548, 427 (1999).

[34] S. Foffa, M. Maggiore, and R. Sturani, Nucl. Phys. B552, 395 (1999).

[35] R. Brustein and R. Madden, Phys. Rev. D 57, 712 (1998).

[36] C. Cartier, E.J. Copeland, and R. Madden, J. High Energy Phys. 01, 035 (2000).

[37] I. Antoniadis, J. Rizos, and K. Tamvakis, Nucl. Phys. B415, 497 (1994).

[38] R. Easther and K.I. Maeda, Phys. Rev. D 54, 7252 (1996).

[39] A. Ghosh, R. Madden, and G. Veneziano, Nucl. Phys. B570, 207 (2000).

[40] R.H. Brandenberger, R. Easther, and J. Maia, J. High Energy Phys. 08, 007 (1998).

[41] D.A. Easson and R.H. Brandenberger, J. High Energy Phys. 09, 003 (1999).

[42] V. Mukhanov and R.H. Brandenberger, Phys. Rev. Lett. 68, 1969 (1992).

[43] R.H. Brandenberger, V. Mukhanov, and A. Sornborger, Phys. Rev. D 48, 1629 (1993).

[44] J.M. Bardeen, P.J. Steinhardt, and M.S. Turner, Phys. Rev. D 28, 679 (1983).

[45] R. Brandenberger and R. Kahn, Phys. Rev. D 29, 2172 (1984).

[46] A.A. Starobinsky, S. Tsujikawa, and J. Yokoyama, Nucl. Phys. B610, 383 (2001). 
[47] C. Cartier, J.C. Hwang, and E.J. Copeland, Phys. Rev. D 64, 103504 (2001).

[48] J.C. Hwang and H. Noh, Phys. Rev. D 61, 043511 (2000).

[49] J.C. Hwang and H. Noh, Phys. Rev. D (to be published), astro$\mathrm{ph} / 0112079$.

[50] A.A. Starobinsky, Pis'ma Zh. Éksp. Teor. Fiz. 30, 719 (1979)
[JETP Lett. 30, 682 (1979)].

[51] D.H. Lyth and E.D. Stewart, Phys. Lett. B 274, 168 (1992).

[52] K. Enqvist and M.S. Sloth, Nucl. Phys. B626, 395 (2002).

[53] T. Moroi and T. Takahashi, Phys. Lett. B 522, 215 (2001).

[54] D.H. Lyth and D. Wands, Phys. Lett. B 524, 5 (2002). 